\title{
Vibrational Signatures of Conformer-Specific Intramolecular Interactions in Protonated Tryptophan
}

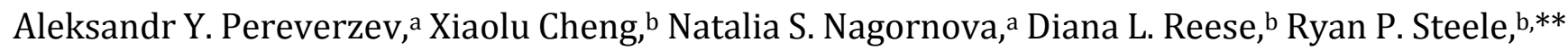
and Oleg V. Boyarkin ${ }^{\mathrm{a}, *}$

aLaboratoire de Chimie Physique Moléculaire, Ecole Polytechnique Federale de Lausanne, EPFL SB ISIC LCPM, Station 6, CH-1015 Lausanne, Switzerland

bDepartment of Chemistry, University of Utah, 315 South 1400 East, Salt Lake City, UT 84112 Henry Eyring Center for Theoretical Chemistry, University of Utah, 315 South 1400 East, Salt Lake City, UT 84112

Corresponding Authors

*Author to whom correspondence should be addressed (experiments). Electronic mail address:

oleg.boiarkin@epfl.ch

**Author to whom correspondence should be addressed (computations). Electronic mail address: ryan.steele@utah.edu 


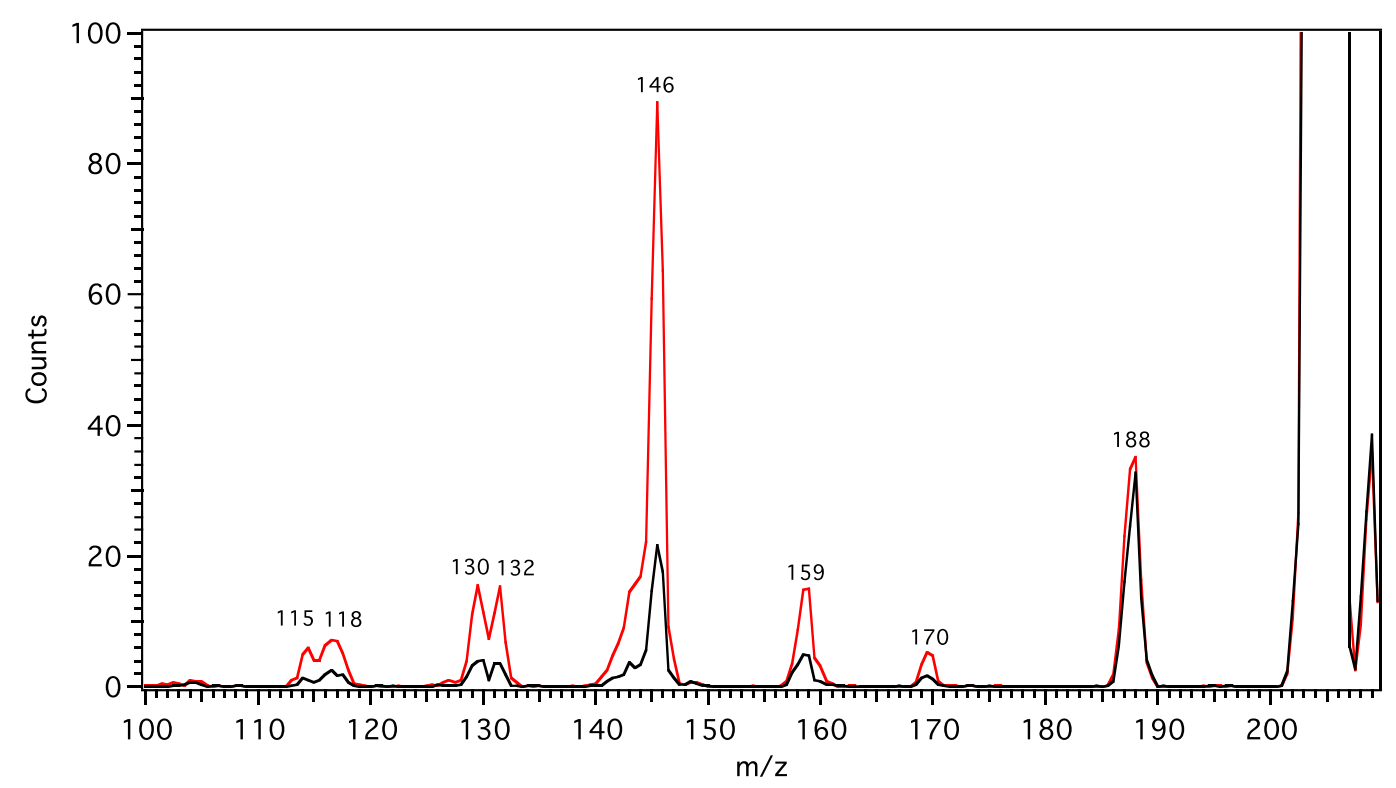

Fig S1. UV photofragmentation mass spectra of cold (black) and IR preheated (red) $\operatorname{TrpH}^{+}$ions. UV wavenumber was fixed at $34400 \mathrm{~cm}^{-1}$, IR wavenumber was fixed at $3339 \mathrm{~cm}^{-1}$.

\section{S2. Peak assignment in $6-\mu \mathrm{m}$ region.}

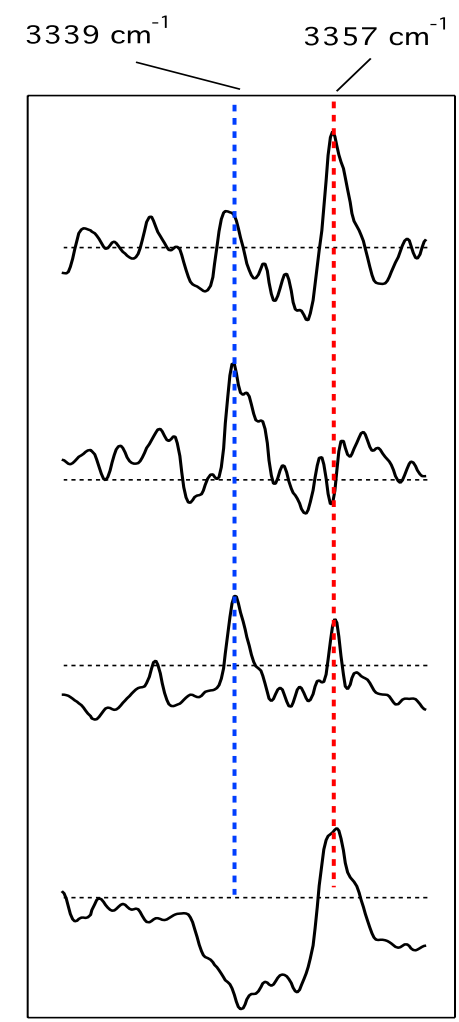

Fig S2. Conformational assignment of peaks labeled 1-4 in Fig. 4a. Each trace shows conformer-specific spectra between 3305 and $3375 \mathrm{~cm}^{-1}$, generated with pump OPO fixed at the corresponding transition in 6- $\mu \mathrm{m}$ region. Assignment is based on comparison of the maximum intensity peak in each trace and the characteristic transitions of conformers $\mathbf{A}$ and $\mathbf{B}$ (blue and red vertical dashed lines, respectively). 
S3. Relative energies of $\operatorname{TrpH}^{+}$isomers, using various levels of theory.

Table S1. Relative energies (kcal/mol) of TrpH+ isomers, using HF.

\begin{tabular}{lccccc}
\hline HF & $\mathbf{6 - 3 1 G ( d , p )}$ & cc-pVDZ & aug-cc-pVDZ & cc-pVTZ & aug-cc-pVTZ \\
\hline A & 0.17 & 0.15 & 0.15 & 0.24 & 0.28 \\
B & 0.29 & 0.35 & 0.64 & 0.71 & 0.79 \\
C & 0.00 & 0.00 & 0.00 & 0.00 & 0.00 \\
D & 2.04 & 2.13 & 2.58 & 2.51 & 2.63 \\
E & 4.30 & 4.29 & 4.48 & 4.56 & 4.62 \\
F & 5.61 & 5.75 & 6.18 & 6.25 & 6.32 \\
G & 4.39 & 4.40 & 4.51 & 4.54 & 4.54 \\
H & 3.56 & 3.61 & 4.09 & 4.26 & 4.34 \\
\hline
\end{tabular}

Table S2. Relative energies (kcal/mol) of TrpH ${ }^{+}$isomers, using density functional M05-2X

\begin{tabular}{lcccc}
\hline M05-2X & 6-31G(d,p) & cc-pVDZ & aug-cc-pVDZ & cc-pVTZ \\
\hline A & 0.09 & 0.28 & 0.00 & 0.00 \\
B & 0.00 & 0.00 & 0.10 & 0.34 \\
C & 0.65 & 0.71 & 0.54 & 0.77 \\
D & 1.38 & 1.49 & 1.98 & 2.07 \\
E & 4.19 & 4.46 & 4.20 & 4.27 \\
F & 4.76 & 5.14 & 5.36 & 5.48 \\
G & 5.24 & 5.48 & 5.23 & 5.45 \\
H & 2.91 & 3.03 & 3.19 & 3.55 \\
\hline
\end{tabular}

Table S3. Relative energies (kcal/mol) of TrpH+ isomers, using density functional B3LYP

\begin{tabular}{lcccc}
\hline B3LYP & 6-31G(d,p) & cc-pVDZ & aug-cc-pVDZ & cc-pVTZ \\
\hline A & 0.02 & 0.01 & 0.001 & 0.04 \\
B & 0.14 & 0.02 & 0.36 & 0.32 \\
C & 0.00 & 0.00 & 0.00 & 0.00 \\
D & 0.87 & 0.82 & 1.63 & 1.53 \\
E & 4.45 & 4.44 & 4.25 & 4.34 \\
F & 4.81 & 4.93 & 5.41 & 5.40 \\
\hline
\end{tabular}




\begin{tabular}{lllll}
\hline $\mathbf{G}$ & 4.48 & 4.60 & 4.38 & 4.41 \\
$\mathbf{H}$ & 3.80 & 3.72 & 4.04 & 4.10 \\
\hline
\end{tabular}

Table S4. Relative energies (kcal/mol) of TrpH+ isomers, using density functional B97M-V

\begin{tabular}{lccc}
\hline B97M-V & 6-31G(d,p) & aug-cc-pVDZ & aug-cc-pVTZ \\
\hline A & 0.78 & 0.45 & 0.46 \\
B & 0.00 & 0.00 & 0.00 \\
C & 0.95 & 0.68 & 0.62 \\
D & 1.14 & 1.42 & 1.45 \\
E & 5.12 & 4.70 & 4.77 \\
F & 5.04 & 5.13 & 5.24 \\
G & 5.52 & 5.14 & 5.15 \\
H & 3.55 & 3.48 & 3.60 \\
\hline
\end{tabular}

Table S5. Relative energies (kcal/mol) of TrpH+ isomers, using RI-MP2

\begin{tabular}{lrrrrc}
\hline RI-MP2 & $\mathbf{6 - 3 1 G ( d , p )}$ & cc-pVDZ & aug-cc-pVDZ & cc-pVTZ & aug-cc-pVTZ \\
\hline A & 1.04 & 0.96 & 0.91 & 0.86 & 0.87 \\
B & 0.00 & 0.00 & 0.00 & 0.00 & 0.00 \\
C & 1.36 & 1.37 & 1.56 & 1.40 & 1.37 \\
D & 1.35 & 1.39 & 1.84 & 1.77 & 1.79 \\
E & 4.70 & 4.65 & 4.58 & 4.82 & 4.74 \\
F & 4.63 & 4.87 & 4.86 & 5.28 & 5.08 \\
G & 5.16 & 5.29 & 5.36 & 5.51 & 5.39 \\
H & 3.08 & 3.10 & 3.02 & 3.47 & 3.27 \\
\hline
\end{tabular}

Table S6. Relative energies (kcal/mol) of $\mathrm{TrpH}^{+}$isomers, using CCSD

\begin{tabular}{llll}
\hline CCSD & cc-pVDZ & $\begin{array}{l}\text { cc-pVDZ } \\
\text { (on RI-MP2/cc-pVTZ structure) }\end{array}$ & $\begin{array}{l}\text { cc-pVTZ } \\
\text { (on RI-MP2/cc-pVTZ structure) }\end{array}$ \\
\hline A & 0.59 & 0.50 & 0.31 \\
B & 0.00 & 0.00 & 0.00 \\
C & 0.80 & 0.76 & 0.61 \\
D & 1.41 & 1.44 & 1.76 \\
E & 4.29 & 4.25 & 4.33 \\
F & 4.99 & 5.02 & 5.42 \\
\hline
\end{tabular}




\begin{tabular}{llll}
\hline $\mathbf{G}$ & 4.79 & 4.74 & 4.81 \\
$\mathbf{H}$ & 3.00 & 3.02 & 3.42 \\
\hline
\end{tabular}

Table S7. Relative energies (kcal/mol) of TrpH+ isomers, using FNO(99\% occ)-CCSD(T)

\begin{tabular}{lll}
\hline FNO-CCSD(T) & $\begin{array}{l}\text { cc-pVTZ } \\
\text { (on CCSD/cc-pVDZ structure) }\end{array}$ & $\begin{array}{l}\text { cc-pVTZ } \\
\text { (on CCSD/cc-pVDZ structure) } \\
\boldsymbol{\Delta M P 2 - c o r r e c t e d ~}\end{array}$ \\
\hline A & 0.25 & 0.51 \\
B & 0.00 & 0.00 \\
C & 0.62 & 0.95 \\
D & 1.72 & 1.64 \\
E & 4.42 & 4.64 \\
F & 5.23 & 5.26 \\
G & 4.88 & 5.13 \\
H & 3.26 & 3.42 \\
\hline
\end{tabular}

\section{S4. Computed anharmonic spectra of $\mathrm{TrpH}^{+}$, using various levels of theory.}

Besides the local-mode, MP2/cc-pVTZ approach, the results of which are reported in the main body of this article, DFT calculations were also performed, and spectra generated using several functionals, including M05-2X, B3LYP, and B97M-V with several basis sets, are shown in Figures S3-S5. Focusing on spacings and relative positions compared with experimental values, MP2/cc-pVTZ performs the best among these methods. Overall, the methods generally come to the same conclusions: the free $\mathrm{O}-\mathrm{H}$ and indole $\mathrm{N}-\mathrm{H}$ stretches are independent of conformer structure; the frequency of free $\mathrm{N}-\mathrm{H}$ in $\mathrm{NH}_{3}$ moiety is lower in conformer $\mathrm{A}$ than that in $\mathrm{B}$, while the frequency of bound N-H is higher in A than that in B. Still, the lowest-frequency N-H stretch is the hardest one to predict in all different methods.

Worth noting is some rather erratic behavior of the M05-2X functional, particularly with large basis sets. The cc-pVTZ results were notably worse than those computed with cc-pVDZ, compared to experiment. Furthermore, we discovered multiple "isomers"-i.e., multiple minima-within the structural isomers presented in the main body of this work. Subtle geometric rearrangements, none of which would be considered new isomers, actually led to distinct minima, which exhibited slightly different harmonic spectra. This behavior was found to be quite sensitive to the 
underlying DFT quadrature grid, and even extremely large grids were not found to be converged. This effect has previously been discussed in N. Mardirossian and M. Head-Gordon, J. Chem. Theor. Comput., 9 4453-4461 (2013).

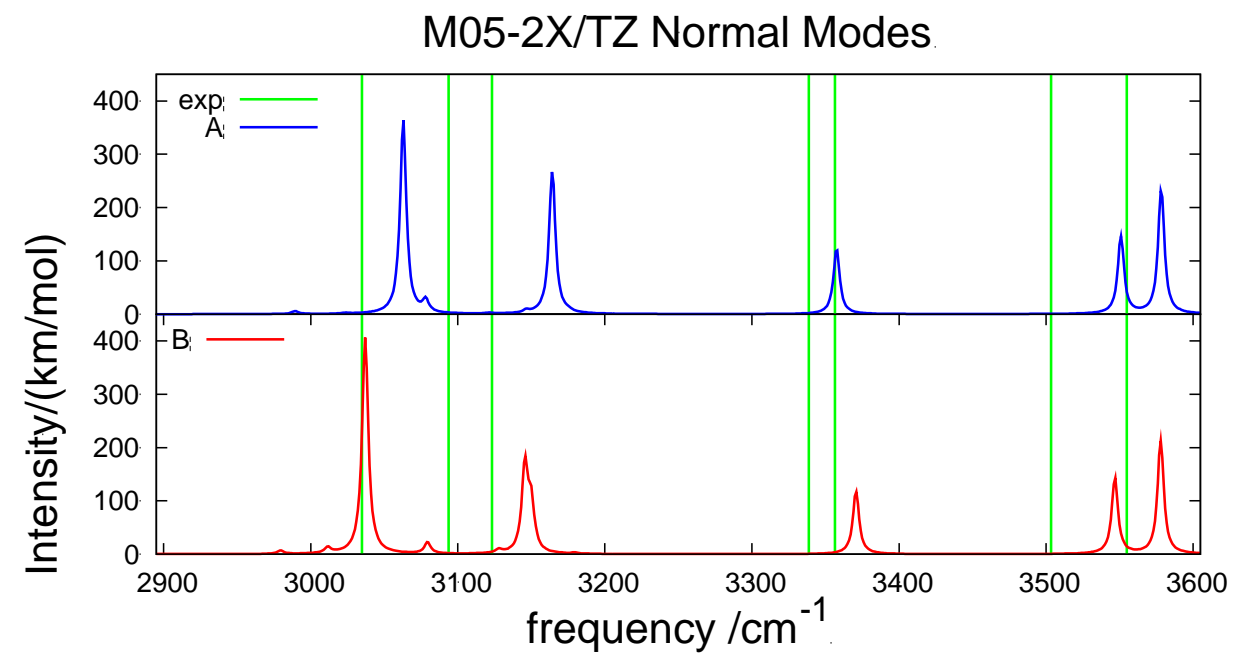

Fig S3. Calculated vibrational spectra of conformer A (blue) and B(red) using density functional M05-2X/cc-pVTZ and normal coordinates, compared with experimental frequencies (green).

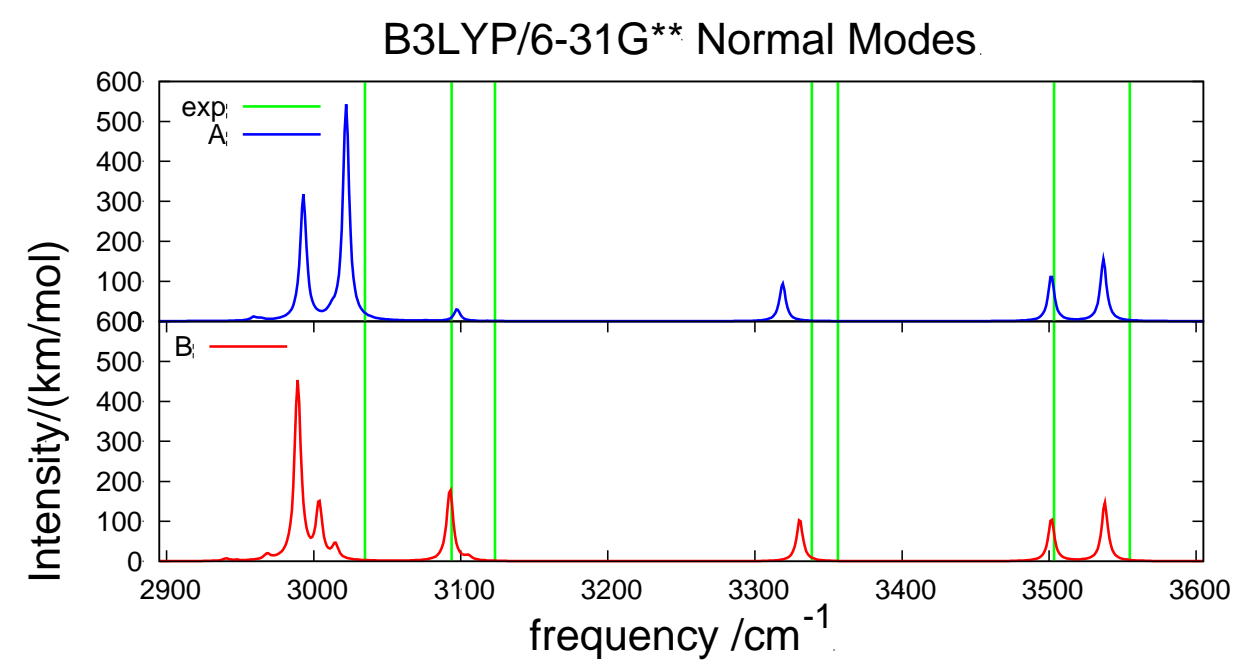

Fig S4. Calculated vibrational spectra of conformer A (blue) and B(red) using density functional B3LYP/6-31G** and normal coordinates, compared with experimental frequencies (green). 


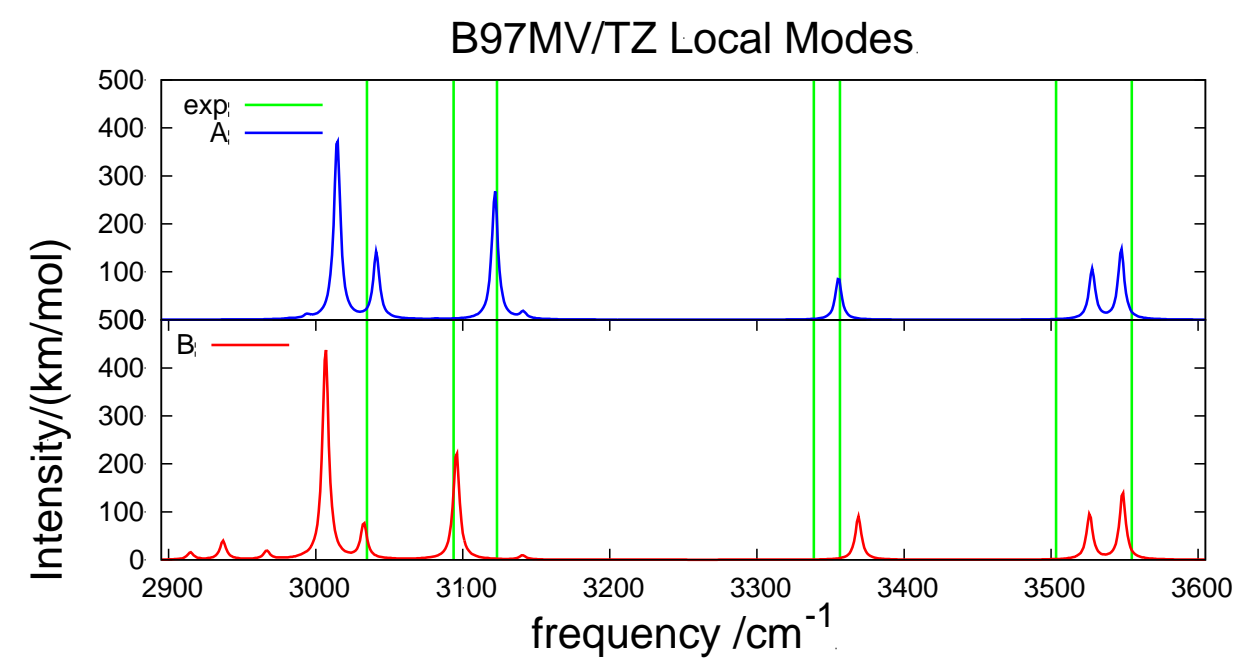

Fig S5. Calculated vibrational spectra of conformer A (blue) and B(red) using density functional B97MV/cc-pVTZ and local-mode coordinates, compared with experimental frequencies (green). Because VSCF is an appropriate theory, a good starting point matters and different sets of rectilinear coordinates can lead to different results. Compared with the local-mode approach (in main body), here we present the spectra of $\mathrm{TrpH}^{+}$using normal modes in Figure S6. At theory level of MP2/cc-pVTZ, for conformer A, the normal mode of peak at $3123 \mathrm{~cm}^{-1}$ is not just $\mathrm{N}-\mathrm{H}$ stretch in $\mathrm{NH}_{3}$, but a combination of that and some $\mathrm{C}-\mathrm{H}$ stretches of indole group, which leads to a triplet instead of one peak in the normal-mode spectra. By localizing this region, we obtain local modes that are all individual X-H stretches of the molecule, which can reduce couplings, especially 3- and higher-order-mode couplings and indeed is a better starting reference.

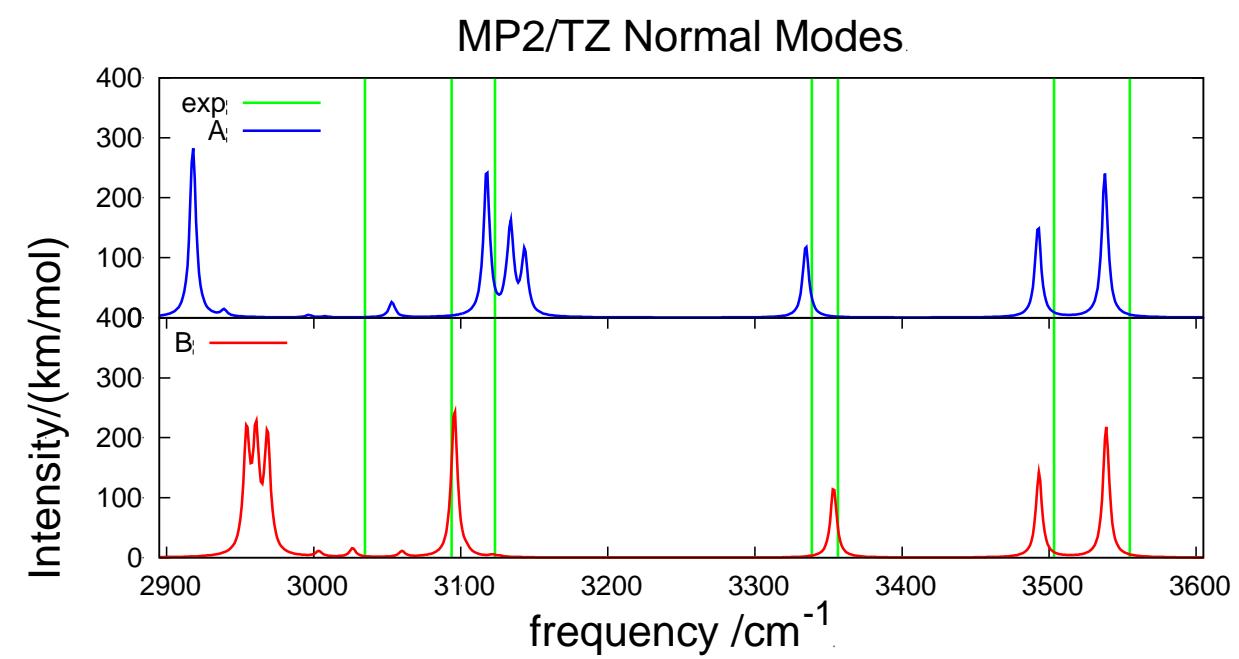

Fig S6. Calculated vibrational spectra of conformer A (blue) and B(red) using dual-basis RIMP2/cc-pVTZ and normal coordinates, compared with experimental frequencies (green).

\section{$\underline{\text { S5. Tests and discussions of the } 3035 \mathrm{~cm}^{-1} \text { peak }}$}

The calculated frequency for this mode is about $50 \mathrm{~cm}^{-1}$ red to the experimental one. To explain this difference we need more investigation of basis set, mode coupling, and vibrational correlation. Focusing first on the basis set, cc-pVTZ is relatively large and likely converged. Even so, we 
calculated the diagonal anharmonic frequency of this mode using MP2 with aug-cc-pVTZ and ccpVQZ. While cc-pVTZ gives $3034.0 \mathrm{~cm}^{-1}$, aug-cc-pVTZ gives $3025.6 \mathrm{~cm}^{-1}$ and cc-pVQZ gives 3031.3 $\mathrm{cm}^{-1}$. Therefore, adopting a basis set larger than cc-pVTZ likely would not result in a large enough blue shift of this peak.

As to mode coupling, we attempted to include 3-mode coupling in the calculation. Again, this is very expensive with the basis set of cc-pVTZ. Therefore, we use cc-pVDZ and include 3-mode couplings that involve this $\mathrm{N}-\mathrm{H}^{+}$stretching mode. The resulting frequency is about $40 \mathrm{~cm}^{-1}$ lower than that from 2-mode representation. Though there are 38 low-frequency modes we did not include, their coupling to the $\mathrm{N}-\mathrm{H}^{+}$stretching is small. We performed 38 2D brute force calculations (with cc-pVDZ) of each low-frequency mode and $\mathrm{N}-\mathrm{H}^{+}$stretching mode, and the biggest difference from the diagonal anharmonic frequency is only $5 \mathrm{~cm}^{-1}$.

The effect of vibrational correlation is hard to determine. VCISD[2] was tried and there is not much difference from results of VDPT2. We also performed 3D brute force calculations of several sets of modes and no significant discrepancies of VDPT2 were discovered. 